
Les enzymes des lysosomes et leur pathologie

On sait que les lysosomes sont des organites subcellulaires bourrés d' enzymes chargées de dégrader de très nombreuses molécules et que le déficit en chacune d'entre elles provoque une maladie de surcharge souvent d'une haute gravité. Lors d'une réunion du Groupe européen d'étude des maladies lysosomales tenue fin septembre 1985 près de Cologne, le point fut fait sur de nombreux problèmes dans un domaine qui représente un modèle de synthèse protéique normale et pathologique. On a analysé de façon fine le trajet que suivent les enzymes après leur biosynthèse, dans le réticulum endoplasmique puis dans l'appareil de Golgi où elles sont glycosylées et acquièrent un groupement mannose-6-phosphate : signal de reconnaissance indispensable pour l'accès aux lysosomes. C'est là qu'elles subissent leur maturation protéolytique finale. Des multiples aspects discutés nous en retiendrons deux particulièrement importants.

I) Les protéines " activateurs" des enzymes. Parmi les enzymes des lysosomes, beaucoup agissent sur des substrats lipidiques. Il semble que cette action ne puisse s'exercer directement, et que les bons offices d'un intermédiaire soient nécessaires. On a isolé des activateurs capables de se lier à l'enzyme d'une part, au substrat, de l'autre, et permettant ainsi la réaction. Un grand nombre d'activateurs a été décrit, tous sont des protéines, mais deux seulement ont été clairement définis, tant par leurs caractères biochimiques que parce que leur déficit donne naissance $a ̀$ des syndromes pathologiques souvent méconnus.

L'activateur des hexosaminidases, décrit dès 1978 par Conzelmann et Sandhoff [I] remplit tous les critères requis: il est spécifique vis-à-vis d'une seule enzyme; son addition à un système contenant de l'hexosaminidase $A$ et le ganglioside GM2 permet l'hydrolyse de ce dernier, dont l'accumulation pathologique provoque la maladie de Tay-Sachs (ou "idiotie amaurotique familiale "). Surtout, son existence est attestée par la pathologie. Il existe en effet deux types biologiques principaux de déficit, celui en hexosaminidase $A$ (variant $B$, maladie de Tay-Sachs proprement dite), et celui qui porte à la fois sur les deux isozymes $A$ et $B$ (variant o ou maladie de Sandhoff). Mais on connaît un type $A B$ qui provoque les mêmes symptômes, où aucun déficit des enzymes elles-mêmes n'est détectable, c'est l'activateur qui est absent et l'addition de cette protéine à des cellules en culture de tels malades "guérit" ces cellules in vitro en permettant à l'hexosaminidase de fonctionner.

Un deuxième activateur a été isolé à l'état pur. Curieusement, sa spécificité est plus large et il active simultanément trois enzymes, l'arylsulfatase $A$, la $\beta$ galactosidase et l' $\alpha$ galactosidase. On connaît en effet des malades chez lesquels cette protéine est absente et dans les tissus desquels les substrats de ces trois enzymes s'accumulent [2]. Cliniquement toutefois, c'est le déficit fonctionnel en arylsulfatase $A$ qui se fait sentir le plus nettement, et les malades ont un tableau clinique de leucodystrophie métachromatique. Mais le gène de cet activateur vient d'être cloné et son locus assigné au chromosome Io [3,4].

2) Anomalies de maturation dans les maladies des lysosomes. Médecine-sciences s'est déjà fait l'écho de maladies génétiques dont le mécanisme met en jeu la maturation des protéines après leur biosynthèse sur les ribosomes. C'est dans les maladies des lysosomes que ces travaux ont été les plus fructueux. Parmi de nombreux exemples possibles, nous prendrons celui de la glycogénose de type II, due au déficit en $\alpha I, 4$ glucosidase ou maltase acide. Elle présente une hétérogénéité clinique avec deux types principaux : une forme infantile rapidement mortelle (maladie de Pompe) et des formes plus tardives comportant des symptômes très divers, le plus souvent de type myopathique. Un travail récent de Reuser et al. portant sur 7 malades [5] a été étendu à 27 cas grâce à une coopération internationale. La méthode comporte l'étude de la biosynthèse de la protéine ou plutôt de ses sous-unités, en fonction du temps dans des cultures de fibroblastes. L'enzyme est extraite, précipitée par des anticorps monospécifiques, puis soumise à une électrophorèse en milieu dissociant et à une autoradiographie. Le marquage se fait le plus souvent par des acides aminés radioactifs mais on peut aussi suivre la glycosylation en employant du mannose radioactif et la fixation du marqueur mannose-6-phosphate par $d u^{32} P$. Le tableau habituel pour l'ensemble des enzymes lysosomales est celui d'un précurseur de grande taille qu'une protéolyse ménagée raccourcit pour aboutir à la sous-unité mature. Dans le cas de l'a glucosidase acide la taille du précurseur est de IIO kilodaltons, celle de l'enzyme mature de 76. L'image est la même, que l'on marque avec un acide aminé, leucine ou méthionine, avec du mannose tritié ou avec $d u{ }^{32} P$. Parmi les malades analysés par le groupe hollandais, pratiquement tous les cas de figure possibles ont été observés, représentant de 5 à 7 formes génétiques distinctes: absence totale de protéine immunoprécipitable; diminution proportionnelle de la quantité du précurseur et de la forme "adulte "; taux normal du précurseur avec diminution ou même absence de maturation; la glycosylation par le mannose n'a jamais été trouvée altérée mais dans plusieurs cas la phosphorylation n'avait pas lieu.

Ce qui semble importer avant tout est la quantité de la forme mature, quelle que soit la production du précurseur. Cette quantité est nulle dans les formes infantiles gravissimes, bien que le précurseur y soit presque toujours présent; elle est faible mais en général détectable dans les formes juvéniles et adultes, en accord avec les dosages d'activité enzymatique chez les malades. Le mécanisme le plus nouveau est l'absence de phosphorylation chez certains sujets qui empêche l'entrée $d u$ précurseur dans le lysosome et sa maturation finale.

Ces données biologiques ont des conséquences cliniques importantes. Plusieurs participants ont insisté sur l'hé- 
térogénéité clinique de nombre de ces maladies, dès lors que le déficit enzymatique n'est pas complet. La maladie est souvent d'apparition trop tardive, trop bénigne - au moins relalivement -, et de symptomatologie trop peu spécifique pour être reconnue par le médecin, et ces formes larvées et tardives sont probablement beaucoup plus fréquentes qu'on ne le pense [6].

J.-C. D.

I. Conzelmann E, Sandhoff K. AB variant of inf antile GM2 gangliosidosis: deficiency of a factor necessary for stimulation of hexosaminidase A-catalyzed degradation of ganglioside $\mathrm{GM}_{2}$ and glycolipid GA2. Proc Natl Acad Sci USA 1978; $75: 3979-83$.

2. Li SC, Kihara $\mathrm{H}$, Scrizawa $\mathrm{S}$, et al. Activator protein required for the enzymatic hydrolysis of cerebroside sulfate. $7 \mathrm{Biol}$ Chem 1985; 260 : I 867-7I.

3. Fujibayashi $\mathrm{S}$, Kao F゙l, Jones $\mathrm{C}$, Morse $\mathrm{H}$ Law M, Wenger DA. Assignment of the gene for human sphingolipid activator protein-2 (SAP-2) to chromosome 10. Am 7 Hum Genet 1985; 37: 741-8.

4. O'Brien JS, Dewji N, Wenger D et al. Molecular cloning of sphingolipid activator protein-1 (SAP-I), the sulfatide sulfatase activator. Molecular genetics $1985 ; 37$ : A I 50.

5. Reuser AJJ, Kroos M, Oude Elfcrink RPJ, Tager JM. Def ects in synthesis, phosphorylation, and maturation of acid $\alpha$-glucosidase in glycogenosis type II. $7 \mathrm{Biol}$ Chem 1985, 260: 8336-42.

6. Tager JM Biosynthesis and deficiency of lysosomal enzymes. Trends in Biological Sciences 1985; 10: $324-5$

\section{Maladie de Hunter chez 2 filles}

Au colloque sur les maladies des lysosomes de Cologne (voir cidessus) Broadhead et Besley (Edimbourg) ont rapporté le cas d'une fillette de 3 ans atteinte de maladie de Hunter. Cette affectation grave, la mucopolysaccharidose type II, qui ressemble cliniquement à la maladie de Hurler, est liée au sexe et donc habituellement ne se manifeste cliniquement que chez les garçons. L'analyse cytogénétique a montré sur un des chromosomes $\mathrm{X}$ une petite délétion en q25, que ne portait aucun de ses parents. La conclusion des auteurs, à la suite d'études de réplication des chromosomes, est que l'X portant la délétion est systématiquement inactivé et que c'est le chromosome apparemment normal, toujours fonctionnel, qui porte le gène pathologique.

Il existe une autre observation, datant de I 983 [I] qui fait état d'une maladie de Hunter chez une fillette de 3 ans porteuse d'une anomalie chromosomique. Il s'agissait d'une translocation apparemment équilibrée, apparue là aussi de novo, entre un des $\mathrm{X}$ et un autosome, le 5. Comme il est habituel dans ce type de translocation, le chromosome $\mathrm{X}$ transloqué sur un autosome est constamment actif et c'est l'autre X qui est inactivé. La scission était localisée en q26, et les auteurs ont conclu qu'elle avait disloqué le locus de l'enzyme déficiente dans la maladie de Hunter, enzyme qui par conséquent n'était plus exprimée. On peut remarquer que cette localisation, encore provisoire, est très proche de la délétion q25 décrite chez le malade dont nous avons parlé plus haut.

Rappelons à cette occasion le grand intérêt des anomalies chromosomiques de l'X dans le sexe féminin. C'est notamment l'analyse de filles atteintes de myopathie de Duchenne de Boulogne et porteuses de translocations, qui a permis de cerner le locus de cette maladie.

J.-C. D.

I. Mossman J, Blunt S, Stephens R, Jones EE, Pembrey M. Hunter's discase in a girl: association with X: 5 chromosomal translocation disrupting the Hunter gene. Arch Dis Child I 983; 58: 911-5

\section{Mous avons I'âge de nos neurones !}

L'ADN des cellules nerveuses ne se renouvelle pas durant la vie. Il est couramment admis que les cellules neuronales ne se divisent pas, et que par conséquent la mort cellulaire, accidentelle ou naturelle, $n$ 'est pas compensée dans le cerveau. Encore était-il possible qu'au moins les cellules vivantes fussent douées d'une importante activité de réparation des dommages qui ne manquent pas de s'accumuler au niveau de l'ADN durant toute une vie.

Un article, publié en mai 1985 dans Science [I] ruine ces espoirs. Il semble bien que non seulement le cerveau ne puisse compter que sur son stock initial de neurones, mais encore que les neurones eux-mêmes ne puissent compter que sur leurs molécules initiales d'ADN.

Pour parvenir à cette conclusion

$m / s n^{\circ}$, vol. 2 jancier $\$$ ()
Slatkin et al. [I] ont utilisé la mesure des isotopes de carbone: le rapport ${ }^{13} \mathrm{C} /{ }^{14} \mathrm{C}$ differe très significativement entre les nourritures européennes et américaines. Si donc on étudie des migrants qui, nés sur un continent, ont vécu presque toute leur vie sur l'autre, ce rapport isotopique dans l'ADN de leurs neurones indiquera si cet $A D N$ a été synthétisé durant la vie embryonnaire et la première enfance (le rapport ${ }^{13} \mathrm{C} /{ }^{14} \mathrm{C}$ sera celui du continent d'origine), ou bien s'il a été progressivement remplacé (le rapport ${ }^{13} \mathrm{C} /{ }^{14} \mathrm{C}$ sera alors celui du continent d'accueil). C'est le $I^{\text {er }}$ résultat qui est indiscutablement observé, démontrant qu'il n'y a pratiquement pas de renouvellement de l' $A D N$ dans les neurones durant toute la vie.

Un autre article également publié en mai 85 dans Nature [2] insistait sur la fréquence et la gravité des remaniements du matériel génétique survenant de façon aléatoire dans les lymphocytes. Puisqu'il y a des chances pour que cela se produise aussi au niveau du cerveau, nous imaginons que des altérations de l'ADN doivent progressivement s'accumuler à ce niveau, facteurs de dysfonctionnement puis de mort cellulaire. Le vieillissement cérébral pourrait bien procéder avant tout de ce mécanisme, redoutable en ce qu'il est difficile d'imaginer ce qui pourrait l'entraver.

A. $\mathrm{K}$.

I. Slatkin DN, Friedman L, Irsa AP, Micca PL. The stability of DNA in human cerebellar neurons. Science 1985; 228: 1002-4.

2. Turner DR, Morley AA, Haliandros M, Kullaca $R$, Sanderson $B J$. In vivo somatic mutations in human lymphocytes frequently result from major gene alterations. Nature $1985 ; 315: 343-5$.

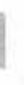

